\title{
Smart communications network management through a synthesis of distributed intelligence and information
}

\author{
J. K. Debenham, S. J. Simoff, J. R. Leaney, and V. Mirchandani
}

\begin{abstract}
Demands on communications networks to support bundled, interdependent communications services (data, voice, video) are increasing in complexity. Smart network management techniques are required to meet this demand. Such management techniques are envisioned to be based on two main technologies: (i) embedded intelligence; and (ii) up-to-the-millisecond delivery of performance information. This paper explores the idea of delivery of intelligent network management as a synthesis of distributed intelligence and information, obtained through information mining of network performance.
\end{abstract}

\section{Introduction}

Communications networks are increasingly becoming integrated with user applications including core business systems. In addition, the core business systems are starting to incorporate highly interactive information-rich Web 2.0 technologies such as different architectures of participation (e.g. weblogs, wikis), peer-to-peer technologies (e.g. Skype, iPhone), many-to-many media publishing platforms (e.g. audio/video podcasts, Web TV, RSS feeds), social bookmarking and other social software [1]. These trends are democratising the creation of value for businesses [2] and provide significant enhancement of our knowledge creation and scientific capabilities. Technologically these trends contribute to the

J. K. Debenham, J. R. Leaney, V. Mirchandani

University of Technology, Sydney, e-mail: \{debenham, johnl, vinodm\}@it.uts.edu.au

S. J. Simoff

University of Western Sydney, e-mail: s.simoff@uws.edu.au

Please use the following format when citing this chapter:

Debenham, J.K., Simoff, S.J., Leaney, J.R. and Mirchandani, V., 2008, in IFIP International Federation for Information Processing, Volume 276; Artificial Intelligence and Practice II; Max Bramer; (Boston: Springer), pp. 415419. 
development of the intelligent ambient habitat [see [3], p.150] of the knowledge civilisation age ${ }^{1}$. As a result, requirements, placed on communication networks are becoming increasingly complex and personalised. These lead to the development of requirements specifications for bundles of interdependent services, often with complex mutual demands and constraints. These services are to be delivered over networks that comprise an increasing variety of heterogeneous network domains each with their differing operational and/or administrative policies. An example of dealing with such bundled, interdependent communications network is the Alcatel-Lucent triple play ${ }^{2}$ architecture [4], which is being provisionally deployed by several Service Providers. This paper explores the idea of delivery of intelligent network management in such networks as a synthesis of distributed intelligence and information, obtained through information mining of network performance.

\section{Network Resource Management}

Network resource management is concerned with the allocation/deallocation of resources to support services that a service provider has committed to a customer. Centralised management approaches cannot cope with increasing management scale, and, there is now a focus on decentralisation and delegation of management decision-making. Service differentiation is a key driver of decentralisation. Products are based on increasing service personalisation, putting more complexity into provisioning processes. Services may be tailored to individual needs, or customers may be provided with seamless access to services across multiple devices or networks without reconfiguration [5]. Furthermore, context- and location-based services allow services to be tailored according to the user's location or interests. Therefore, the trend is moving towards more frequent and complex management activities, with per-customer or per-subscriber management. This trend is already evident in existing and next-generation broadband architectures, where per-subscriber policies are required [6]. Service differentiation is also driving more dynamic and adaptive management systems. The network needs to be reconfigured, depending on the user's environment and the available network resources. This is especially the case with respect to business-critical services that demand "five nines" availability (i.e. $99.999 \%$ which equates to only 5 minutes of downtime a year). Timely, adaptive and flexible management is necessary for dynamically changing network environments, for the introduction of new services on-demand [7], as well to evolve to accommodate changing user and Service Provider requirements.

\footnotetext{
${ }^{1}$ The term "knowledge civilization age is borrowed from 3. Wierzbicki, A.P., Nakamori, Y.: Creative Space: Models of Creative Processes for the Knowledge Civilization Age. Springer, Berlin/Heidelberg (2006)

2 "Triple play" refers to voice, video and data services over an Internet Protocol (IP) based network.
} 
Autonomic computing, which requires systems to be more self-managing, adaptive and aware of their environment demonstrates some potential to address network resource management issues, but there will need to be several enhancements - greater communication and negotiation, improved trustworthiness, visibility and accountability, and evolved intelligence [8].

A simpler approach is Management by Delegation (MbD), where management intelligence is shifted closer to the managed systems through Service Level Agreements (SLAs). These protocols are used for automated service negotiation. They include: (i) the dynamic service negotiation protocol (DSNP) for service level negotiation using a client-server architecture, and service level specification (SLS) negotiation at the IP layer; and (ii) the 'resource negotiation and pricing protocol' (RNAP) protocol allows the negotiation of prices for the contracted services. DSNP suffers from the drawbacks of a centralized architecture. RNAP has limited scalability because it relies on periodic signalling from subscribers to negotiated services. These and other protocols, presented in details in [9] contain useful ideas but are not capable of negotiating bundles of inter-dependent services from multiple service providers simultaneously.

\section{Agent-based negotiation and Information mining in Communications Systems and Networks}

The deployment of intelligent agents in communications systems has been widely investigated since the early-1990s. Sadly the potential has not yet been fully realised. What we believe to be the reason for this failure is also the central assumption of our approach: that the way to deliver intelligent network management is through a synthesis of distributed intelligence and as accurate as possible information about the performance of the network. Despite failing to deliver this vision, thirteen years later interest in agents in the communications industry continues to flourish. The eMarkets Group ${ }^{1}$ has developed the QDINE ${ }^{2}$ approach, which adopts a distributed open market approach to service management. Service charging is described within an SLA, allowing the use of any charging model appropriate to a provided service. The synthesis of information and decision-making has led the researchers from the group to merge information mining with intelligent agency in an on-going sequence of works on "Information-based Agency" [e.g. see [10, 11]]. The unifying theory underpinning this work is information theory. The deployment of this synthesis to communications systems is starting to gain interest [12]. Given the fundamental significance of information theory to communications, intelligent agents based on information theory seem to be the natural choice of technology for coupling

\footnotetext{
${ }^{1}$ http://research.it.uts.edu.au/emarkets

${ }^{2}$ The QDINE site is: http://qdine.it.uts.edu.au/. The public section of the site includes lists of the publications where one can find the details of the formal specification language for describing SLAs together with its ontology.
} 
intelligence with data- and information mining in communications networks. Earlier work in the application of data mining in telecommunications has focused on fault isolation and forecasting telecommunication equipment failures based on mining communication network data, which describes the state of the hardware and software components in the network. These approaches, however, did not have embedded mechanisms to recognise and take in account various changes in communications networks and their predictive models are acceptable only over a limited period of time [13].

\section{The Elements of the Smart Communications Network Management Technology}

The elements of the proposed technological framework for smart communications network management that addresses the above discussed issues include:

- $\quad$ Language and ontologies for formal representation of SLAs about bundles of interdependent services from multiple service providers and an SLA Negotiation Framework that utilises these formal representations;

- Algorithms for mining network predictive models and short-term network performance information, which provide the necessary information about the trends and local variations in the network behaviour - this information can be used both during the negotiation of what is possible to deliver, i.e. the values of the different SLA parameters, and deliver what has been agreed, i.e. the strategies that enable the fulfilment of what the SLA promises.

- Strategies for flexible resource provisioning that enable bundling the 'best' mix of services based on the current data and flexible adapting of the service provider to the actual situation on the net in order to meet its obligation in the SLA;

- Mechanisms that enable informed routing, which rely on predictive models of network performance and by up-to-the-millisecond information correction before the actual routing of the packages is performed.

\section{Conclusions}

The synthesis of distributed intelligence and information, obtained through information mining of network performance has the potential to advance network resource management. Presented ideas address network performance management are looking at reducing the gap between current network performance, and, desired network performance, using artificial intelligence techniques which have been demonstrated to be flexible, and to scale. The challenge in this is to design a 
system that operates quickly and does not place significant overhead on the network.

\section{References}

1. ISTAG: New Business Sectors in Information and Communication Technologies: The Content Sector as a case study. Information Society Technology Advisory Group (ISTAG) (2007)

2. Tapscott, D., Williams, A.D.: Wikinomics: How Mass Collaboration Changes Everything. Portfolio, London (2006)

3. Wierzbicki, A.P., Nakamori, Y.: Creative Space: Models of Creative Processes for the Knowledge Civilization Age. Springer, Berlin/Heidelberg (2006)

4. Kompella, V.: A Novel architecture for Triple Play services. Proceedings Asia Pacific Regional Internet Conference on Operational Technologies APRICOT 2005, Kyoto, Japan (2005)

5. Falcarin, P.: A CPL to Java compiler for dynamic service personalization in JAINSIP server. IEC Annual Review of Communications 57 (2004)

6. Anschutz, T.: DSL evolution - architecture requirements for the support of QoSEnabled IP Services. DSL Forum Technical Report TR-059. Architecture and Transport Working Group (2003)

7. Sloman, M., Lupu, E.: Policy specification for Programmable Networks. Proceedings of the International Workshop on Active Networks, Berlin, Germany (1999)

8. Strassner, J.: Autonomic networking: Theory and practice. 9th IFIP/IEEE International Symposium on Integrated Network Management, IM05 (2005) 786 786

9. Sarangan, V., Chen, J.-C.: Comparative study of protocols for Dynamic Service Negotiation in the next-generation Internet. IEEE Communications Magazine (2006) 151-156

10. Zhang, D., Simoff, S.J., Debenham, J.K.: Exchange rate modelling for enegotiators using text mining techniques. In: Lu, J., Ruan, D., Zhang, G. (eds.): EService Intelligence - Methodologies, Technologies and Applications. Springer, Heidelberg (2007) 191-211

11. Sierra, C., Debenham, J.: Information-Based Agency. Procedings of the 20th International Joint Conferences on Artificial Intelligence IJCAI07, Hyderabad, India (2007) 1513-1518

12. Rocha-Mier, L.E., Sheremetov, L., Batyrshin, I.: Intelligent agents for real time data mining in telecommunications networks. In: Gorodetsky, V., Zhang, C., Skormin, V.A., Cao, L. (eds.): Autonomous Intelligent Systems: Multi-Agents and Data Mining. Springer-Verlag (2007) 138-152

13. Weiss, G.M.: Data mining in the Telecommunications industry. In: Wang, J. (ed.): Encyclopedia of Data Warehousing and Mining. Information Science Publishing (2008) 